\title{
Surgical Intervention for Uterine Fibroids. Our 4-Year Experience and Literature Review: Is It Time to Centralise Care Provision Via Specialist Fibroid Centres?
}

\author{
SOPHIE MICHELLE STRONG ${ }^{1}$, MICHAIL SIDERIS ${ }^{2}$, ZWELIHLE MAGAMA $^{1}$, \\ SCHARAZED ROUABHI ${ }^{1}$ and FUNLAYO ODEJINMI ${ }^{1}$ \\ ${ }^{1}$ Whipps Cross University Hospital, Barts Health NHS Trust, London, U.K.; \\ ${ }^{2}$ Women's Health Research Unit, Queen Mary University of London, London, U.K.
}

\begin{abstract}
Background: Leiomyomas are common in women of reproductive age, for whom treatment-stratification can be challenging. We assessed factors influencing pre-operative selection of surgical intervention and compare outcomes. Patients and Methods: A literature review was performed and surgical interventions for fibroids at a London hospital (20152018) were retrospectively examined. Outcomes assessed were estimated blood loss, length of stay (LOS) and complications. Data were analysed using univariate non-parametric inferential statistics. Results: A total of 258 cases were identified. Estimated blood loss was statistically significantly lower for laparoscopic versus open myomectomy [200 (interquartile range; $I Q R)=100-200 \mathrm{vs} .400(I Q R=200-700$ $\mathrm{ml}), \quad p<0.001]$. Length of stay was also statistically significantly lower in the laparoscopic cohort compared with open hysterectomy [1 (IQR=1-1) days vs. $2(I Q R=2-3$ days $)]$. Similar results were noted when comparing laparoscopic versus open hysterectomy [167 $(I Q R=100-200)$ vs. 500 $(I Q R=100-750) \mathrm{ml}, \mathrm{p}<0.001]$. No differences in complications were reported across groups. Patients counselled by a surgeon trained in minimally invasive surgery (MIS) opted more frequently for laparoscopy $(p<0.01$, rho=-0.669). Estimated blood loss during MIS was $225 \mathrm{ml}$ versus $545 \mathrm{ml}$ for non-MIS, and $285 \mathrm{ml}$ for the overall cohort $(p<0.01)$.The length of stay was statistically significantly lower for those treated with MIS (1.37 days) versus other surgeons (2.65 days), or overall cohort (1.63 days) $(p<0.01$ for all associations). Conclusion:
\end{abstract}

This article is freely accessible online.

Correspondence to: Dr Sophie Michelle Strong, Whipps Cross university Hospital, Whipps Cross Road, Leytonstone, London, E11 1NR, UK. E-mail: sophiestrong@nhs.net

Key Words: Fibroid surgery, myomectomy, centralised care, fibroid centres.
Laparoscopic surgery offers superior short-term outcomes when performed by experienced operators. Multidisciplinary counselling and treatment stratification should be goldstandard practice. Centralisation of care provision in fibroid Centres of Excellence is required.

Uterine fibroids are the most common solid benign tumours in women of reproductive age affecting over $70 \%$ of women by menopausal age (1). Associated symptoms such as menorrhagia, dysmenorrhea, pressure effects and subfertility can have an impact quality of life. One third of women with fibroids request treatment for symptom management (2), however, many of them are dissatisfied with the information received during counselling and are often unaware of the short- and long-term outcomes at the units where they receive treatment. Besides the financial and emotional burden to the individual who suffers from the effects of fibroids (3), there is also a societal burden. In the UK, surgical management of fibroids carries a significant financial burden to the United Kingdom National Health Service (NHS). Recent costs for the NHS tariff were estimated at $£ 2,400$ for uterine artery embolization, $£ 2,609$ for myomectomy and $£ 3,275$ for hysterectomy. Of the 31,624 hysterectomies performed in England in 2017 and 2018, 60\% $(18,974)$ were for fibroids, and the total in-patient cost was estimated at $£ 103,568,600$. It is also estimated that 1,600 myomectomies are performed in the UK each year (4). In the United States of America, the annual cost of uterine fibroid treatment is estimated at between $\$ 5.9$ and 34.4 billion (5).

Despite acknowledgement of the burden of uterine fibroids and the fact that most women who require interventions for fibroids will have surgery, there are few high-quality data to formulate evidence-based guidelines (6) and thus decision making in such cases can be challenging and is not always based on published evidence. There is, however, an overall consensus from emerging studies to suggest that minimal access surgery offers benefits over traditional open surgery for myomectomy (7), and hysterectomy (8). 
Technical challenges associated with fibroid surgery have been extensively discussed in the literature (9). Limiting surgical complications is essential for improving patient safety and patient experience and also reducing cost for healthcare systems. This dictates an emerging need for women to be managed in centres where all treatment options are available with data supporting clinical outcomes, enabling informed consent. This view is clearly outlined by the All-Party Parliamentary Group on Women's Health in "Informed choice? Giving women control of their healthcare" (10). The document suggests an agreed best practice pathway which ensures that the right women receive the correct information and treatment. Previous large database studies have investigated characteristics of patients who undergo interventions for uterine fibroids and noted differences in characteristics based on race and age (11). In light of recent issues with morcellation, units have been encouraged to publish data on outcomes of management of uterine fibroids (12), albeit only for laparoscopic interventions, and to date most publications have not focused on all surgical interventions for fibroids.

We thus performed a structured mini-review of the literature to assess existing evidence. We then reviewed all of the surgical interventions for fibroids offered locally in a busy East London teaching hospital. We aimed to assess factors that influence selection of surgical intervention; this included demographics of our local population, symptoms at presentation, operator experience and pre-operative clinical assessment and imaging. We also aimed to compare intraoperative and short term post-operative outcomes across the different groups of surgical interventions.

\section{Patients and Methods}

Structured mini-review of the literature. Initially we performed a structured mini-review of the literature on MEDLINE. We aimed to identify all the studies which included women who underwent surgical interventions for fibroids and which reported any short- or long-term outcomes following surgery, from January 2010 to September 2019. We focused on those studies that reported comparative outcomes between Minimally Invasive versus open Surgery and specified expertise of surgeon performing the operation. The search strategy is outlined: "FIBROID*" AND (myomectomy OR "hysterectomy" OR "hysteroscopic myomectomy" OR "transcervical resection of FIBROID*" OR "open myomectomy" OR "laparoscopic myomectomy" OR "open hysterectomy" OR "laparoscopic hysterectomy") filtered from 1/1/2010 to September 2019; no language restriction was applied. The conclusions of the studies which fit the above criteria, and deemed as influential, were taken into consideration as comparative evidence.

Hospital data. We designed a retrospective study protocol based on Population, Intervention, Comparisons and Outcomes (PICO). We included all women who underwent myomectomy (open and laparoscopic), Transcervical resection of fibroid (TCRF) or hysterectomy for fibroids between March 2015 and November 2018. All cases were identified retrospectively from the electronic patient records. Surgical intervention was discussed and decided based upon the severity and nature of clinical symptoms, ultrasound/magnetic resonance imaging findings where applicable, operator experience, and patients' wishes. All cases were managed according to the national as well as the local guidelines for gold standard practice. Following a relevant Food and Drug Administration warning, all women were offered patient information leaflets and consent forms specifically for morcellation. Despite acknowledgement of the risks women still opted to undergo minimal access procedures.

Data extraction. Data were extracted retrospectively in pre-designed spreadsheets. This included operating surgeon's details, demographics [age, parity, body mass index (BMI) and ethnicity], presenting symptoms, clinical assessment and pre-operative imaging including ultrasound parameters (size/location of largest fibroid and number of fibroids), or magnetic resonance imaging. Data were also collected on alternative medical treatments that had been offered prior to surgery. Operating surgeon's details were collected from the electronic patient records.

With regards to intra-operative findings, we collected data on the size and location of the largest fibroid, presence of endometriosis and whether the cavity was breached. Data on histopathology were also collected post-operatively.

The estimated blood loss including drop in the haemoglobin $(\mathrm{Hb})$ level, duration of the surgical procedure, any reported complications, length of stay and need for re-admission were defined as outcomes.

All data were collected by three independent authors (SS/SR, SS/ZM)

Data analysis. Inferential univariate non-parametric statistics were used to describe our data; analysis was performed on IBM SPSS for Macintosh (Version 25.0; IBM Corp., Armonk, NY, USA). Normality of data distributions was assessed with Shapiro-Wilks test. Based on the nature of the data, associations were explored using Spearman's rho and medians were compared using KruskalWallis and Mann-Whitney tests.

Ethics approval. Ethical approval for the study was sought from the local research unit, however, as it was an audit of quality of care provision, formal ethics approval was not deemed necessary.

\section{Results}

Literature review. Our search yielded 1,176 titles; nine of these were applicable to our study. From those studies, three were performed at tertiary centres (13-15), and six at unspecified or local settings (7, 16-20). Six studies supported MIS as gold standard modality which offers better short- and long-term outcomes post-surgery $(7,13,15-18)$. Two studies supported centralised care as the way forward to improve fibroid surgery outcomes, minimise cost and improve safety $(15,18)$. Those studies were taken into consideration when formulating conclusions in the discussion section.

Hospital data. A total of 258 cases were identified; the median age was 42 years [interquartile range (IQR) $=37-46$ years] and the median body mass index was $28(\mathrm{IQR}=24-32) \mathrm{kg} / \mathrm{m}^{2}$. Almost half of women who were operated on were Afro- 
Caribbean in origin $(109 ; 44.7 \%)$. Table I summarises patient demographics, symptoms at presentation, and pre-operative ultrasound reports. Fifty-eight women were offered and opted for open myomectomy $(22.5 \%)$, 93 for laparoscopic myomectomy (36\%), five for open total abdominal hysterectomy (1.9\%), 29 for total laparoscopic hysterectomy $(11.2 \%)$ and 30 for laparoscopic subtotal hysterectomy (11.6\%). Overall, 59/64 (92.1\%) of the women who underwent hysterectomy had a laparoscopic procedure. Forty-three women had a TCRF (16.7\%).

Procedure-specific demographics, intraoperative findings, perioperative and post-operative outcomes. Women who opted for hysterectomy were on average older than those who underwent myomectomy [50 (IQR=46-51) vs. 37 (IQR=3241) years; $p<0.001)$ ]; the laparoscopic myomectomy cohort were the youngest women [37 (IQR=32-41) years]. Body mass index was similar across the different procedure groups $(p>0.05)$ (Table II).

Of the women who underwent myomectomy, 58/151 $(61.5 \%)$ had a laparoscopic procedure. The median number, weight and size of the largest fibroid was 6 ( IQR $=1.75$ 13.75), $1035 \mathrm{~g}(\mathrm{IQR}=580-1436 \mathrm{~g})$ and $10 \mathrm{~cm}(\mathrm{IQR}=8-15 \mathrm{em})$ respectively for open myomectomy versus 3 ( $\mathrm{IQR}=1-5.5)$, $126 \mathrm{~g}(\mathrm{IQR}=50-295 \mathrm{~g})$ and $14 \mathrm{~cm}(\mathrm{IQR}=12-16 \mathrm{~cm})$ for laparoscopic myomectomy ( $p<0.001$ for all comparisons).

Blood loss was statistically significantly lower $(p<0.001)$ for laparoscopic myomectomy [200 $\mathrm{ml}(\mathrm{IQR}=100-200 \mathrm{ml})]$ versus open myomectomy [400 $\mathrm{ml}(\mathrm{IQR}=200-700 \mathrm{ml})]$, as it was for laparoscopic versus open hysterectomy $(167 \mathrm{ml}$ $(\mathrm{IQR}=100-200 \mathrm{ml}) v s .500 \mathrm{ml}(\mathrm{IQR}=100-750 \mathrm{ml}) ; p<0.001]$. In the laparoscopic myomectomy cohort $(\mathrm{N}=93)$, there was one bowel injury in a patient known to have dense bowel adhesions from previous surgery (managed by extending the suprapubic incision, exteriorising the bowel with primary anastomosis) and two red blood cell transfusions compared with one bladder injury, one transfusion and one readmission for the open myomectomy group ( $\mathrm{N}=58)$.

Histopathological results confirmed there was no malignancy across all groups of intervention. This was in keeping with pre-operative and the intra-operative findings supporting safety of morcellation in appropriately selected women.

The length of stay (LOS) was statistically significantly lower for laparoscopic myomectomy (1 day, 1-1) versus open myomectomy ( 2 days, $2-3$ ) (rho $=-0.428, p<0.01)$; as expected TCRF led to the shortest stay, and all women attended as day cases.

Treatment stratification and post-operative outcomes. Of the 91 nulliparous women, 60 were offered and accepted laparoscopic myomectomy $(p<0.01)$. BMI did not affect treatment selection or post-operative outcomes including
Table I. Cohort demographics \& clinical symptoms at presentation.

\begin{tabular}{|c|c|}
\hline \multicolumn{2}{|l|}{ Characteristic } \\
\hline \multicolumn{2}{|l|}{ Age } \\
\hline Median (IQR) & $42(37-46)$ \\
\hline \multicolumn{2}{|l|}{ BMI } \\
\hline Median (IQR) & $28(24-32)$ \\
\hline \multicolumn{2}{|l|}{ Parity } \\
\hline Median (IQR) & $1(0-2)$ \\
\hline \multicolumn{2}{|l|}{ Ethnicity, n (\%) } \\
\hline Afro-Caribbean & $109(44.7 \%)$ \\
\hline White & $68(27.9 \%)$ \\
\hline Asian & $47(19.3 \%)$ \\
\hline Other/mixed & $20(8.2 \%)$ \\
\hline \multicolumn{2}{|l|}{ Clinical symptoms, n (\%) } \\
\hline Menorrhagia/pain & $88(43.8 \%)$ \\
\hline Menorrhagia & $78(38.8 \%)$ \\
\hline Infertility & $24(11.9 \%)$ \\
\hline Pain & $11(5.5 \%)$ \\
\hline \multicolumn{2}{|c|}{ Clinical size of uterus, weeks of gestation } \\
\hline Median (IQR) & $16(12-20)$ \\
\hline \multicolumn{2}{|l|}{ Number of fibroids* } \\
\hline Median (IQR) & $3(2-7)$ \\
\hline \multicolumn{2}{|l|}{ Size of largest fibroid* } \\
\hline Median (IQR) & $6(5-8)$ \\
\hline \multicolumn{2}{|l|}{ Location of fibroids, $\mathrm{n}(\%)^{*}$} \\
\hline SubserosaI & $61(35.3 \%)$ \\
\hline Intramural & $46(26.6 \%)$ \\
\hline SubmucosaI & $29(16.8 \%)$ \\
\hline Mixed subserosal/intramural & $13(7.5 \%)$ \\
\hline Pedunculated & $13(7.5 \%)$ \\
\hline Broad ligament & $7(4 \%)$ \\
\hline Cervical & $4(2.3 \%)$ \\
\hline \multicolumn{2}{|l|}{ Pre-operative imaging, n (\%) } \\
\hline US scan prior to intervention & $236(100 \%)$ \\
\hline \multicolumn{2}{|l|}{ Need for additional MRI mapping } \\
\hline Yes & $18(25.4 \%)$ \\
\hline No & $53(74.6 \%)$ \\
\hline \multicolumn{2}{|l|}{ Procedure performed, n (\%) } \\
\hline \multicolumn{2}{|l|}{ Myomectomy } \\
\hline Open & $58(36 \%)$ \\
\hline Laparoscopic & $93(61.5 \%)$ \\
\hline Total & 151 \\
\hline \multicolumn{2}{|l|}{ Hysterectomy } \\
\hline Open & $5 / 64(7.8 \%)$ \\
\hline Laparoscopic & $29 / 64(45.3 \%)$ \\
\hline Laparoscopic-assisted subtotal & $30 / 64(46.8 \%)$ \\
\hline Total laparoscopic & $59 / 64(92.1 \%)$ \\
\hline $\mathrm{TCRF} /$ Total procedures & $43 / 258(16.7 \%)$ \\
\hline
\end{tabular}

BMI: Body mass index; IQR: interquartile range; MRI: magnetic resonance imaging; US: ultrasound; TCRF: transcervical resection of fibroids. *By US.

blood loss, duration of procedure or LOS $(p>0.01)$. Younger women tended to have shorter $\operatorname{LOS}$ ( $\mathrm{rho}=-0.190, p=0.003$ ). Afro-Caribbean women had a higher blood loss on average (341 ml) compared to Asian $(216 \mathrm{ml})$ and Caucasian women $(183 \mathrm{ml})(p=0.003)$. 


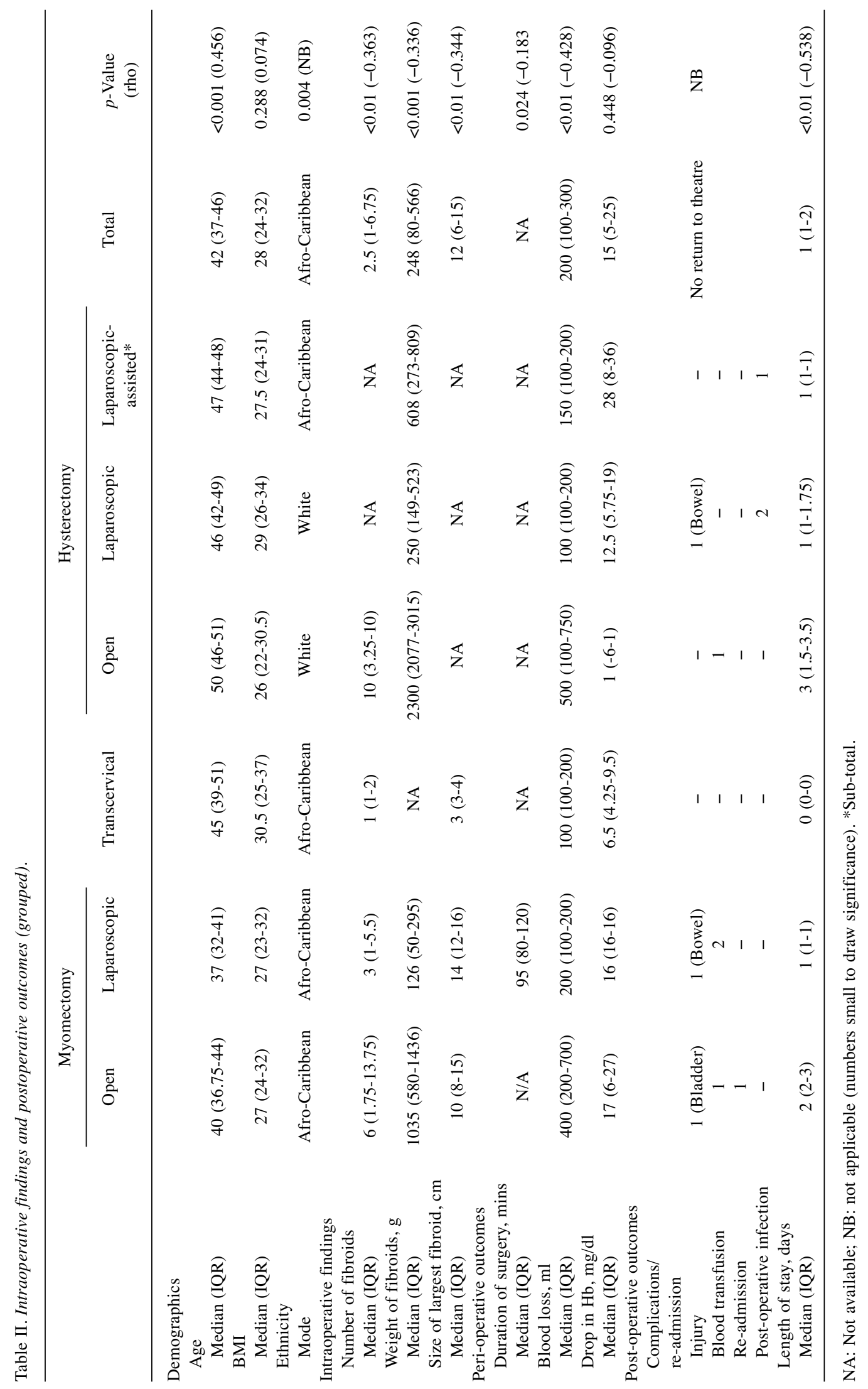


Operator experience seemed to directly affect treatment selection; patients counselled by a senior surgeon trained in MIS (FO) seem to opt for MIS (rho=-0.669, $p<0.01$ ). The average blood loss for procedures performed by the senior MIS surgeon was 225 versus $545 \mathrm{ml}$ for non-MIS trained surgeons and $285 \mathrm{ml}$ for the overall cohort $(p<0.01)$. Similarly, the LOS was statistically significantly lower (1.37vs. $2.65 v s .1 .63$ days, respectively; $p<0.01$ for all associations).

As expected, increased clinical size of the uterus and the size of the largest fibroid on ultrasound increased blood loss, LOS and duration of surgery ( $p<0.01$ for all associations). Increased clinical size of uterus also affected treatment selection, favouring open to laparoscopic surgery.

\section{Discussion}

Literature review. The vast majority of evidence was found to support minimal access as a gold standard approach to improving fibroid surgery. Laparoscopy offers shorter recovery time with better post-operative outcomes. Many studies support multidisciplinary approach to treatment stratification as the way forward to improve safety, patient experience and post-operative outcomes. Several studies also support operator experience as a major factor contributing to safe operating, better training of juniors and smoother running of the service. Those arguments underline the potential need for centralisation of care provision in fibroid centres as has been supported directly by other studies (21).

Our literature search also revealed there were no studies that included hysterectomy, myomectomy and TCRF collectively. To our knowledge, our study is the first to review all these modalities and therefore will allow women to clearly look at all surgical approaches in one place rather than relying on systematic reviews with variable surgical experience of the studies included in the review.

Hospital data. Over two thirds of the women included in our cohort offered and opted for laparoscopic versus laparotomic myomectomy. Similarly, when a hysterectomy was indicated, over $90 \%$ of women chose a laparoscopic approach as opposed to open surgery. Our data show that laparoscopic surgery seems to be in more demand from younger and nulliparous women, and that most women would take up this approach when minimal access surgery is offered.

Our study reaffirms previously published outcome data in support of minimal access interventions for the management of uterine fibroids. This included less blood loss, shorter LOS and fewer complications; this subsequently creates the argument of less cost to healthcare systems.

Despite a trend towards open myomectomy in the case of a clinically or ultrasound confirmed larger uterus, it seems that BMI did not affect the decision regarding laparoscopy. Further to this, the overall size of fibroids operated via laparotomic operations was higher in the case of the surgeon trained in MIS compared to the other the surgeons in this study. We also found that such a surgeon achieves reduction in LOS without incurring additional complications. We also confirmed there was no malignancy in the histopathological results for all patients. Our results support a recent review supporting the safety of morcellation in appropriately selected women (22).

Clinical interpretation. Our study shows that where available, there is a clear trend that patients would opt for laparoscopic treatment; the question is do they always get that option? Although indications for fibroid surgery follow well-established guidelines, there is still limited evidence with regard to patient selection for laparoscopic versus laparotomic myomectomy. Therefore, there is still a discrepancy behind access to technically challenging laparoscopic surgery, which we see from our experience locally, is dependent on operator's proficiency and experience to perform such procedures. Undoubtedly, the size of fibroids and of the uterus in general, can present a true technical surgical challenge.

Our literature review $(7,9,11)$ showed a paucity of data for all fibroid interventions in single publications and that inferences were made on the basis of systematic reviews with the limitation of not taking surgical expertise into account. This presents a strong argument for a standardised evidence-based multidisciplinary approach, in other words, a fibroid-treatment pathway based on the highest quality evidence available.

Finally, we acknowledge the fact that MIS requires advanced training and unfortunately this could imply extra cost to healthcare services. Essentially, a proficient operator is on average faster than a novice one (23), therefore, under the guidance of an experienced surgeon at a Fibroid Centre, opportunities could be generated for trainees to learn these technical skills in a timely manner and in a safe environment. This necessitates the formation of a supportive network, which includes both good quality supervision, as well as provision of a satellite simulation-based facility for optimising the learning curve. In a nutshell, training the next generation of surgeons expert in MIS means that more women will be offered laparoscopic treatment of fibroids in the future and receive better care provision.

Recommendations: An argument for centralisation of care provision. We noted that women prefer laparoscopic surgery, which seems to be safer in safe hands with better short-term outcomes. This is a well-established argument, but there is a series of challenges. Which women should be offered laparoscopic surgery, and which of those would definitely benefit more? How is the volume of cases to be managed? Despite reduced LOS and hence cost, laparoscopic 
procedures can be equally costly, especially for prolonged operations where theatre lists are full; how should this be addressed? Finally, MIS has a steep learning curve and training the future generation can be a challenge. Healthcare systems are continuously pressured to meet targets, and this means that training shifts to service provision to compensate for these needs.

The way to address this is by creating hubs of excellence; in other words, fibroid treatment centres. Centralising care means optimising time and money investment towards building an expert multidisciplinary team. Such centres can bring experts together and be the stepping-stone to prospectively establishing well-supported guidelines. This can be either on a regional or even national scale and can potentially reduce the current 'grey zones' on decision making. Further to this argument, agreeing on a gold standard practice guideline involves not only retrospective evaluation of available evidence and expert opinion, but also a novel framework of core performance outcomes, which can guarantee better reporting and hence quality of future evidence. And this has been the example of the Core Outcomes in Women's and Newborn Health (CROWN) initiative, aiming to harmonise core outcomes in most areas of obstetrics and gynaecology (24).

Creating fibroid treatment centres of excellence can also result in better provision of high-class training both for specialist registrars, as well as for consultants who want to develop proficiency in advanced laparoscopy. The great advantage of such initiatives is the provision of safe training with no compromise of service, and hence no additional cost to the healthcare system overall. In simple terms, more surgeons can train in MIS in a safe environment, at no extra cost.

Strengths of the study. A major strength of the study is review of the available evidence prior to interpreting our results. Regarding our patient cohort, it had a wide range of age, BMI and parity. Coming from an East London Hospital, our patients represent an extremely diverse high-risk population, which could be therefore be applied to any other high-risk population in the UK or worldwide. We also followed a retrospective protocol for this study. Compared to other published work, we had a considerable sample size, which allows us to draw valid conclusions. Further to this, our team comprised a good mix of surgeons highly experienced and advanced in MIS with generalist obstetricians and gynaecologists, which is a valid representation of an average teaching centre in the UK.

Limitations. Our study was limited by its retrospective nature which occasionally made it difficult to extract certain data due to the observed heterogeneity in the way data were reported. Most of the procedures were carried out by or under the supervision of a single surgeon who may have had a predilection for MIS even in potentially difficult cases, however, this in itself may represent a strength as there was no increase in complication rates. A further limitation is the use of this single population and therefore univariate analysis and not multivariate analysis, which could restrict the generalisability of our results.

\section{Conclusion}

Laparoscopic surgery is safe and seems to offer superior short-term outcomes for young women who opt to undergo surgical intervention for fibroids. Creating fibroid treatment centres of excellence can harmonise care provision as decision making will be standardised based on robust guidelines and more women could be offered and benefit from laparoscopic surgery. As proven by other examples, centralising care reduces overall costs. Finally, fibroid treatment centres can be a step forward to safely train the new generation of surgeons in MIS and at no additional cost, increasing both the number technically proficient as well as their experience at a more junior stage of training.

\section{Conflicts of interest}

None declared.

\section{Authors' Contributions}

The primary Author Sophie Michelle Strong drafted the article with Michail Sideris. Zwelihle Magama, Scharazed Rouabhi and Sophie Michelle Strong performed the data collection. Michail Sideris performed statistical analysis of the results. Funlayo Odejinmi was the senior Author who conceived the study and methodology, and edited the article. Funlayo Odejinmi was also the senior surgeon who carried out all of the laparoscopic procedures.

\section{References}

1 Boosz AS, Reimer P, Matzko M, Romer T and Muller A: The conservative and interventional treatment of fibroids. Dtsch Arztebl Int 111(51-52): 877-883, 2014. PMID: 25597366. DOI: 10.3238/arztebl.2014.0877

2 Donnez J and Dolmans MM: Uterine fibroid management: From the present to the future. Hum Reprod Update 22(6): 665-686, 2016. PMID: 27466209. DOI: 10.1093/humupd/dmw023

3 Brito LG, Panobianco MS, Sabino-de-Freitas MM, Barbosa Hde F, de Azevedo GD, Brito LM and Candido-Dos-Reis FJ: Uterine leiomyoma: Understanding the impact of symptoms on womens' lives. Reprod Health 11(1): 10, 2014. PMID: 24476380. DOI: 10.1186/1742-4755-11-10

4 Statistics HE: Hospital admitted patient care activity, 2017-18. Hospital Admitted Patient Care Activity, 2018. Available from: https://digital.nhs.uk/data-and-information/publications/ statistical/hospital-admitted-patient-care-activity/2017-18

5 Cardozo ER, Clark AD, Banks NK, Henne MB, Stegmann BJ and Segars JH: The estimated annual cost of uterine leiomyomata in the United States. Am J Obstet Gynecol 206(3): 211 e211-219, 2012. PMID: 22244472. DOI: 10.1016/j.ajog.2011.12.002 
6 Al-Hendy A, Myers ER and Stewart E: Uterine fibroids: Burden and unmet medical need. Semin Reprod Med 35(6): 473-480, 2017. PMID: 29100234. DOI: 10.1055/s-0037-1607264

7 Bhave Chittawar P, Franik S, Pouwer AW and Farquhar C: Minimally invasive surgical techniques versus open myomectomy for uterine fibroids. Cochrane Database Syst Rev 10: CD004638, 2014. DOI: 10.1002/14651858.CD004638.pub3

8 Aarts JW, Nieboer TE, Johnson N, Tavender E, Garry R, Mol BW and Kluivers KB: Surgical approach to hysterectomy for benign gynaecological disease. Cochrane Database Syst Rev 8: CD003677, 2015. DOI: 10.1002/14651858.CD003677.pub5

9 Havryliuk Y, Setton R, Carlow JJ and Shaktman BD: Symptomatic fibroid management: Systematic review of the literature. JSLS 21(3), 2017. PMID: 28951653. DOI: 10.4293/JSLS.2017.00041

10 HEALTH A-PPGOWS: Informed choice? Giving women control of their healthcare. All-party parliamentary group on women's health, 2017. Available from: http://www.appgwomens health.org/news/2017/3/27/the-all-party-parliamentary-group-onwomens-health-report-launched

11 Borah BJ, Laughlin-Tommaso SK, Myers ER, Yao X and Stewart EA: Association between patient characteristics and treatment procedure among patients with uterine leiomyomas. Obstet Gynecol 127(1): 67-77, 2016. PMID: 26646122. DOI: 10.1097/AOG.0000000000001160

12 FDA: Quantitative assessment of the prevalence of unsuspected uterine sarcoma in women undergoing treatment of uterine fibroids. Food and Drug Administration Reports, 2014. Available from: https://www.fda.gov/media/88703/download

13 Mallick $\mathrm{R}$ and Odejinmi F: Pushing the boundaries of laparoscopic myomectomy: A comparative analysis of perioperative outcomes in 323 women undergoing laparoscopic myomectomy in a tertiary referral centre. Gynecol Surg 14(1): 22, 2017. PMID: 29200989. DOI: 10.1186/s10397-017-1025-1

14 Bean EM, Cutner A, Holland T, Vashisht A, Jurkovic D and Saridogan E: Laparoscopic myomectomy: A single-center retrospective review of 514 patients. J Minim Invasive Gynecol 24(3): 485-493, 2017. PMID: 28104498. DOI: 10.1016/j.jmig. 2017.01 .008

15 Tan N, McClure TD, Tarnay C, Johnson MT, Lu DS and Raman SS: Women seeking second opinion for symptomatic uterine leiomyoma: Role of comprehensive fibroid center. J Ther Ultrasound 2: 3, 2014. PMID: 25512867. DOI: 10.1186/20505736-2-3

16 Marin-Buck A, Karaman E, Amer-Cuenca JJ, Lison JF, Torok P, Karaaslan O, Valenti G, Zito G, Biondi A and Chiofalo B: Minimally invasive myomectomy: An overview on the surgical approaches and a comparison with mini-laparotomy. J Invest Surg: 1-8, 2019. PMID: 31322011. DOI: 10.1080/08941939.2019. 1642422
17 Cianci S, Gueli Alletti S, Rumolo V, Rosati A, Rossitto C, Cosentino F, Turco LC, Vizzielli G, Fagotti A, Gallotta V, Ciccarone F, Scambia G and Uccella S: Total laparoscopic hysterectomy for enlarged uteri: Factors associated with the rate of conversion to open surgery. J Obstet Gynaecol 39(6): 805-810, 2019. PMID: 31001998. DOI: 10.1080/01443615.2019.1575342

18 Hartmann KE, Fonnesbeck C, Surawicz T, Krishnaswami S, Andrews JC, Wilson JE, Velez-Edwards D, Kugley S and Sathe NA: Management of uterine fibroids. Rockville (MD): Agency for Healthcare Research and Quality (US). Comparative Effectiveness Review, No. 195, 2017. PMID: 30789683.

19 Leung M, Murji A, Allaire C, Singh SS, Thiel J, Tulandi T and Shore EM: Factors influencing the difficulty of laparoscopic myomectomy: The development of a surgical rating tool. Eur $\mathbf{J}$ Obstet Gynecol Reprod Biol 231: 230-234, 2018. PMID: 30439651. DOI: 10.1016/j.ejogrb.2018.10.047

20 Bretschneider CE, Frazzini Padilla P, Das D, Jelovsek JE and Unger CA: The impact of surgeon volume on perioperative adverse events in women undergoing minimally invasive hysterectomy for the large uterus. Am J Obstet Gynecol 219(5): 490 e491-490 e498, 2018. PMID: 30222939. DOI: 10.1016/j.ajog.2018.09.003

21 Olufowobi O, Sharif K, Papaionnou S, Neelakantan D, Mohammed $\mathrm{H}$ and Afnan $\mathrm{M}$ : Are the anticipated benefits of myomectomy achieved in women of reproductive age? A 5-year review of the results at a UK tertiary hospital. J Obstet Gynaecol 24(4): 434-440, 2004. PMID: 15203587 . DOI: 10.1080/01443610410001685600

22 Odejinmi F, Aref-Adib M, Liou N, Sideris M and Mallick R: Rethinking the issue of power morcellation of uterine fibroids: Is morcellation the real problem or is this another symptom of disparity in healthcare provision? In Vivo 33(5): 1393-1401, 2019. PMID: 31471384 . DOI: 10.21873/invivo.11616

23 Sideris M, Papalois A, Tsoulfas G, Majumder S, Toutouzas K, Koletsis E, Dedeilias P, Lymperopoulos N, Papagrigoriadis S, Papalois V and Zografos G: Developing an international combined applied surgical science and wet lab simulation course as an undergraduate teaching model. Biomed Res Int 2015: 463987, 2015. PMID: 26613083. DOI: 10.1155/2015/463987

24 Duffy J, Rolph R, Gale C, Hirsch M, Khan KS, Ziebland S, McManus RJ and International Collaboration to Harmonise Outcomes in Pre-eclampsia (iHOPE): Core outcome sets in women's and newborn health: A systematic review. BJOG 124(10): 1481-1489, 2017. PMID: 28421657. DOI: 10.1111/14710528.14694
Received December 8, 2019

Revised December 22, 2019

Accepted December 30, 2019 